\title{
An improved multiplex PCR for diagnosis and differentiation of Mycoplasma hyopneumoniae and Mycoplasma hyorhinis
}

\author{
Abhijit K. Barate', Hwi-Young Lee ${ }^{1}$, Hye-Won Jeong ${ }^{1}$, Lam Quang Truong ${ }^{1}$, \\ Hong-Gu Joo ${ }^{2}$, Tae-Wook Hahn ${ }^{1, *}$ \\ ${ }^{1}$ Department of Veterinary Medicine, College of Veterinary Medicine \& Institute of Veterinary Science, \\ Kangwon National University, Chuncheon 200-701, Korea \\ ${ }^{2}$ Department of Veterinary Medicine, College of Veterinary Medicine, Jeju National University, Jeju 690-756, Korea \\ (Received: August 22, 2011; Revised: January 31, 2012; Accepted: February 1, 2012)
}

\begin{abstract}
A multiplex PCR was developed for the simultaneous detection and differentiation of Mycoplasma (M.) hyopneumoniae and M. hyorhinis in clinical samples. Improved sensitivity is advantage of this technique over the previously reported multiplex assay. It was capable of detecting as little as $125 \mathrm{fg}$ genomic DNA from M. hyopneumoniae and $62.5 \mathrm{fg}$ genomic DNA from M. hyorhinis. Application of this multiplex PCR method to field isolates showed that M. hyopneumoniae and M. hyorhinis were present in 29\% (107 of 370) of lung specimens and no mycoplasmas were detected in $56 \%$ (208 of 370 ) of the slaughtered pigs' lungs. At the farm level, M. hyopneumoniae and M. hyorhinis were detected in 34 of $36(94.4 \%)$ randomly selected farms. We conclude that this assay would prove itself a value tool for monitoring these mycoplasmal infections and both M. hyopneumoniae and M. hyorhinis have been widely spread in swine herds of Korea.
\end{abstract}

Keywords: enzootic swine pneumonia, multiplex PCR, Mycoplasma

\section{Introduction}

Mycoplasma (M.) hyopneumoniae (Mhp) causes mycoplasmal pneumonia in pigs. In combination with other opportunistic pathogens, it is involved in the development of enzootic pneumonia (EP) and porcine respiratory disease complex (PRDC) [16, 20]. Recently, it has been reported that M. hyorhinis (Mhr) is also capable of eliciting EP in swine [13]. Both of these species are able to grow in serum-supplemented Friis media, and the easily grown Mhr can limit the isolation of Mhp [11, 15]. In the past years, serology [3, 8], fluorescent probes [4] and multiplex PCR (mPCR) [13, 17] have been utilized for specific identification of these mycoplasma species. The mPCR, a single reaction PCR, is quick and can be easily adopted in laboratories. It is preferred over serology, fluorescent probes, PCR, nested PCR as it specifically identifies and distinguishes the two mycoplasmas in one step [12, 13, 17]. However, in the reported mPCR assays the sizes of the amplified fragments are very different and large [13, 17]. It is generally accepted that in PCR, efficiently amplified loci negatively influence the yield at other loci. Moreover, smaller fragments tend to be more efficiently amplified than the larger fragments [23]. Ideally in a PCR, the size of the PCR amplicons should be approximately $100 \mathrm{bp}$ different, so that they can be easily differentiated using usual gel electrophoresis. For instance, the assays with larger amplicons will have lower sensitivity, and their combination with smaller amplicons may give false negative results. Keeping these issues in mind we designed an MPCR having smaller PCR products with 84-bp size difference. Thus, one might expect to detect the two organisms more efficiently and with reduced risk of false negative results. Therefore, objectives of the current study were 1) to develop a mPCR to overcome the problem associated with efficiently amplified loci; 2) to evaluate the sensitivity of mPCR for detection of Mhp and Mhr; and 3) to apply this mPCR to lung lesion samples from Korean slaughtered pigs to assess the

\footnotetext{
*Corresponding author

Tel: +82-33-250-8671, Fax: +82-33-244-2367

E-mail: twhahn@kangwon.ac.kr
} 
presence of Mhp and Mhr and to correlate the results with previous reports.

\section{Materials and Methods}

\section{Mycoplasma strains and growth conditions}

The reference strains used in this study were Mhp J (ATCC 25934) and Mhr (ATCC 27717). Field strains were isolated from the lung lesions of slaughter pigs from 36 herds. The strains were cultivated in Friis broth, and cultures showing a gradual color shift from red to yellow were used in the mPCR.

\section{DNA extraction}

Genomic DNA from the reference strains and field isolates was extracted using alkaline lysis method [17]. For which, $1.5 \mathrm{~mL}$ of the broth cultures was centrifuged and re-suspended in $50 \mu \mathrm{L}$ of lysis buffer $(0.25 \%$ SDS in $50 \mathrm{mM} \mathrm{NaOH}$ ). After heating for $5 \mathrm{~min}$ at $95^{\circ} \mathrm{C}$, the mixtures were cooled, and the supernatants from a subsequent centrifugation step were used as the templates for mPCR. DNA quantifications were done using NanoDrop 1000 Spectrophotometer (Thermo Scientific, USA).

\section{Primers and $\mathrm{mPCR}$ reaction}

Primers (Bioneer, Korea) MhpF-1 (5'-ACTAGATAG GAAATGCTCTAG-3') and MhpR-2 (5'-ATACTACTC AGGCGGATCATTTAAC-3') were used for the amplification of 16S rRNA gene from Mhp [13], and the primers MhrF-3 (5'-GTAGTCAAGCAAGAGGATGT-3') and MhrR-4 (5'-GCTGGAGTTATTATACCAGGA-3') were used for the amplification of the p37 gene from Mhr [6]. Based on these sequences, the theoretical amplification products are $430 \mathrm{bp}$ (Mhp) and $346 \mathrm{bp}$ (Mhr) in length. The amplifications were performed in $25 \mu \mathrm{L}$ reaction mixtures containing $0.8 \mathrm{U}$ of recombinant GoTaq DNA polymerase (Promega, USA), 1X Green GoTaq Flexi buffer, $2 \mathrm{mM} \mathrm{MgCl} 2,0.2 \mathrm{mM}$ of each dNTP, and $20 \mu \mathrm{M}$ of each forward and reverse primer. Thirty-five cycles $\left(1 \mathrm{~min}\right.$ at $94^{\circ} \mathrm{C} ; 1 \mathrm{~min}$ at $45^{\circ} \mathrm{C}$; and $1 \mathrm{~min}$ at $72^{\circ} \mathrm{C}$ ) were run in a P708 Thermal cycler (ASTEC, Japan). Amplified PCR products $(10 \mu \mathrm{L})$ were analyzed in $1.8 \%$ agarose gel and visualized after staining with ethidium bromide.

\section{DNA sequencing}

The PCR products were cleaned up using HiYield Gel/ PCR DNA fragment extraction kit (RBC Bioscience,

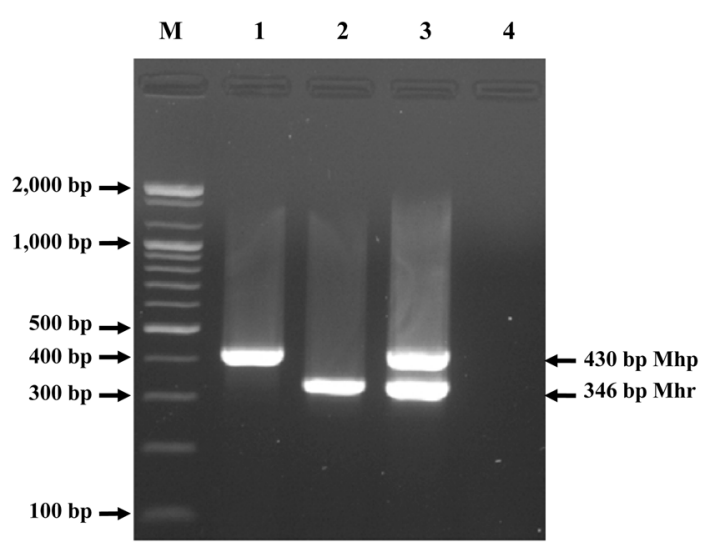

Fig. 1. Electrophoretic profile of specific amplicons from 16s RNA (Mhp) and the p37 gene (Mhr). Lane M: 100bp ladder, Lane 1: Mhp J ATCC 25934, Lane 2: Mhr ATCC27717, Lane 3: Mhp J ATCC 25934 and Mhr ATCC 27717, Lane 4: Negative control.

Taiwan). Sequencing was performed on both strands at sequencing service (Bioneer, Korea). Finally, sequence alignment was carried out using SerialCloner (ver. 2.1; SerialBasics, France) and Basic Local Alignment Search Tool (BLAST) algorithm [1].

\section{Results}

\section{Multiplex PCR assay and its specificity}

Application of this mPCR to the genomic DNA from Mhp and Mhr (3ng each) produced the expected size bands, which were distinguishable on a $1.8 \%$ agarose gel (Fig. 1, lanes 1-3). Primer pairs MhpF-1 and MhpR-1 successfully amplified a 430-bp DNA fragment from the Mhp 16S rDNA gene. Primer pairs MhrF-3 and MhrR4 successfully amplified a 346-bp DNA fragment from the p37 gene of Mhr. The control reaction which lacks the template DNA, did not exhibit any amplification. No cross reaction were obtained between the two mycoplasma species indicating good specificity of the assay.

Every mPCR assay produced the expected 430-bp band (Mhp) and 346-bp band (Mhr). Non-specific bands were not detected. DNA sequences of the mPCR products (Fig. 1, lane 3) were aligned using Serial Cloner and BLAST searched for confirming the presence of specific amplicons. The 430-bp amplicon matched $100 \%$ with the $16 \mathrm{~S}$ rRNA gene from Mhp (GeneBank accession no. Y00149.1), and the 346-bp 


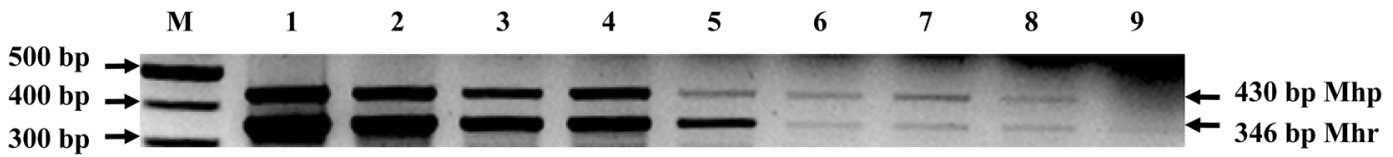

Fig. 2. Sensitivity of mPCR in detecting serial dilutions of genomic DNA using specific primer pairs for 16s RNA (Mhp) and the p37 gene (Mhr). Lane M: 100-bp ladder, Lane1-9: mPCR performed with Mhp ATCC 25934 and Mhr ATCC27717 each at ng-pg concentrations. Lane 1: $1 \mathrm{ng}$, Lane 2: $100 \mathrm{pg}$, Lane 3: $10 \mathrm{pg}$, Lane 4: $5 \mathrm{pg}$, Lane 5: 1 pg, Lane 6: $0.5 \mathrm{pg}$, Lane 7: $0.25 \mathrm{pg}$, Lane 8: $0.125 \mathrm{pg}$, Lane 9: $0.0625 \mathrm{pg}$.

matched $100 \%$ with the $\mathrm{p} 37$ gene from Mhr (GeneBank accession no. CP002170.1). Note that all the BLAST hits for the $\mathrm{p} 37$ amplicon sequence were Mhr sequences. Interestingly, the 346-bp band demonstrated two nucleotide difference with Mhr p37 gene (GeneBank accession no. CP002669.1) and four nucleotide difference with Mhr p37 gene (GeneBank accession no. X14140.1). These results suggest that natural variation exists in Mhr p37 gene. Furthermore, the specificity of the primers was determined by using the BLAST search. The BLAST search revealed that the primers were specific for the species examined. Even though MhpR showed homology with Mhr, no non-specific product was observed from this organism due to lack of complementarily between MhpF and Mhr. Other BLAST hits were not known to infect pigs, and the matches with high $\mathrm{E}$ values were not considered.

\section{Sensitivity}

To determine the detection limits of this assay, serial dilutions of genomic DNA from each mycoplasma species were prepared using laboratory grade water. These diluted samples were then tested using mPCR as described above (Fig. 2). The mPCR could detect as little as $125 \mathrm{fg}$ genomic DNA from Mhp and $65 \mathrm{fg}$ genomic DNA from Mhr. Considering the genome size of these mycoplasma theoretically this corresponds to 100 organisms for Mhp and 70 organisms for Mhr [14, $17,19]$. Thus this assay was found to be more sensitive than previously reported mPCR [17] in detecting Mhp (125 fg vs. 1 pg, respectively) and Mhr (65 fg vs. 500 fg, respectively) simultaneously.

Isolation is considered as gold standard for detection of porcine mycoplasma. However, this method is expensive and has many limitations such as requirement for specialized media, serum that is negative for antibodies against mycoplasma, time consuming (4 8 weeks) and contamination [9]. Furthermore, ELISA is commonly used to determine the mycoplasma infection
Table 1. Incidence of Mycoplasma (M.) in the lung lesions of Korean slaughtered pigs

\begin{tabular}{lc}
\hline \hline & $\begin{array}{c}\text { No. of Isolates } \\
(\%)\end{array}$ \\
\hline M. hyopnuemoniae & $53(14)$ \\
M. hyorhinis & $36(10)$ \\
Co-infection of M. hyopnuemoniae & $18(5)$ \\
and M. hyorhinis & $55(15)$ \\
Negative on PCR & $208(56)$ \\
No Mycoplasma species & $370(100)$ \\
Total & \\
\hline
\end{tabular}

Table 2. Incidence of Mycoplasma infection in Korean pig farms

\begin{tabular}{lc}
\hline \hline & No. of farms (\%) \\
\hline Mycoplasma species & \\
(M. hyopnuemoniae, M. hyorhinis, & \\
co-infection of M. hyopnuemoniae & $34(94.4)$ \\
and M. hyorhinis) & \\
No Mycoplasma species & $2(5.6)$ \\
Total & $36(100)$ \\
\hline
\end{tabular}

in herd. However, this method also has disadvantages like interference by maternal antibodies in young animals, low sensitivity, variable antibody response and longer time required by animals for seroconversion (6 $\sim 9$ weeks) $[2,20,21]$. The current $\mathrm{mPCR}$ assay will help to detect Mhp and Mhr at low cost, high sensitivity and short time (1 2 day).

\section{Isolation and detection of Mhp and Mhr}

Finally, the field samples were tested with $\mathrm{mPCR}$ to investigate the distribution of these mycoplasmas in Korean pig farms. Infection of Mhp and Mhr was detected in $107(29 \%)$ of the 370 lungs lesion samples. Using this method, 53 isolates (14\%) were identified as Mhp, 36 isolates $(10 \%)$ were identified as Mhr, 18 isolates (5\%) were identified as the co-infection of Mhp and Mhr, and 
55 isolates (15\%) were negative with $\mathrm{mPCR}$ (Table 1). At the farm level, either Mhp or Mhr were detected at 34 farms (94.4\%), but no positive reaction was obtained in 2 out of 36 farms (5.6\%) (Table 2).

\section{Discussion}

EP is a chronic respiratory disease affecting pigs with all ages. In combination with other opportunistic or respiratory pathogens, Mhp can cause PDRC [16], and Mhr has been implicated in the development of lung lesions caused by porcine reproductive and respiratory syndrome virus [10]. Serum-supplemented Friss media is used to isolate these organisms. Co-existence of Mhr and M. flocculare is known to hinder the growth of Mhp in culture media, thus complicating diagnosis. Serology $[3,8]$, fluorescent probes [4] and mPCR [13, 17] have each previously been utilized for the specific identification of mycoplasma species. Differentiation based on serology is hampered by the cross-reaction between these two species. Moreover, western blotting is costly and requires longer detection times [8]. In situ hybridization (ISH) is highly specific, but it can only be applied on necropsy samples, which prevents ISH's use for the surveillance of live animals. Also, tissue processing can obscure and hinder the sensitivity of ISH. mPCR has recently been used to differentiate these organisms. It is less expensive, rapid and easy to perform.

The primer pairs used in this study were reported previously, and the specificity of the MPCR is guaranteed $[6,13]$. The specificity of the MPCR was also evaluated by DNA sequencing and BLAST search, which indicated that these primers are specific for these mycoplasma species. The sensitivity test showed that this assay is highly sensitive. Quantities as small as $125 \mathrm{fg}$ genomic DNA from Mhp and $65 \mathrm{fg}$ genomic DNA from Mhr could be detected using this multiplex reaction. Using nested PCR, a more sensitive detection of Mhp alone was reported by some earlier studies [12, 18, 22]. However the nested PCR is a two-stage process and thus requires more time and reagents. Also owing to high sensitivity, the nested PCR is more likely to produce false positive results with slightest environmental contamination. Given that Mhp and Mhr can both cause EP, the techniques allowing simultaneous detection of both these organisms are more desirable. Notably, the sensitivity of our mPCR is similar to one nested PCR reported earlier (80 Mhp organisms) [5]. Thus advantage of our assay over the earlier PCR techniques is rapidity and simultaneous detection of both primary pathogens causing EP at lower numbers (100 CFU of Mhp and $70 \mathrm{CFU}$ of Mhr).

The incidence rate of Mhp including co-infection of Mhp and Mhr in Korean slaughtered pigs (19\%) is similar to the previous findings on prevalence of Mhp (20.1\%) [7]. Also, either Mhp or Mhr were detected on 34 (94.4\%) of the Korean swine farms. These findings indicate that Mhp and Mhr have been spread widely over majority of farms in Korea and underscore the need for efficient control measures. In conclusion, the results of this study show that our mPCR is highly sensitive and specific. Because of its higher detection ability, this assay may be very useful for assessment of clinical samples and early implementation of the preventive measures.

\section{Acknowledgments}

This research was supported by Technology Development Program for Agriculture and Forestry, Ministry for Food, Agriculture, Forestry and Fisheries and was supported technically by Institute of Veterinary Science, Kangwon National University, Korea.

\section{References}

1. Altschul SF, Gish W, Miller W, Myers EW, Lipman DJ. Basic local alignment search tool. J Mol Biol 1990, 215, 403-410.

2. Andreasen M, Nielsen JP, Baekbo P, Willeberg P, Bøtner A. A longitudinal study of serological patterns of respiratory infections in nine infected Danish swine herds. Prev Vet Med 2000, 45, 221-235.

3. Bölske G, Strandberg ML, Bergström K, Johansson KE. Species-specific antigens of Mycoplasma hyopneumoniae and cross-reactions with other porcine mycoplasmas. Curr Microbiol 1987, 15, 233-239.

4. Boye M, Jensen TK, Ahrens P, Hagedorn-Olsen T, Friis NF. In situ hybridisation for identification and differentiation of Mycoplasma hyopneumoniae, Mycoplasma hyosynoviae and Mycoplasma hyorhinis in formalin-fixed porcine tissue sections. APMIS 2001, 109, 656-664.

5. Calsamiglia M, Pijoan C, Trigo A. Application of a nested polymerase chain reaction assay to detect 
Mycoplasma hyopneumoniae from nasal swabs. J Vet Diagn Invest 1999, 11, 246-251.

6. Caron J, Ouardani M, Dea S. Diagnosis and differentiation of Mycoplasma hyopneumoniae and Mycoplasma hyorhinis infections in pigs by PCR amplification of the p36 and p46 genes. J Clin Microbiol 2000, 38, 1390-1396.

7. Chu KS, Kang MS, Jo YS, Lee JW. Detection of porcine circovirus 2, porcine reproductive and respiratory syndrome virus and Mycoplasma hyopneumoniae from swine lungs with lesions by PCR. Korean J Vet Ser 2008, 31, 71-77.

8. Freeman MJ, Armstrong CH, Sands-Freeman LL, Lopez-Osuna M. Serological cross-reactivity of porcine reference antisera to Mycoplasma hyopneumoniae, $M$. flocculare, $M$. hyorhinis and $M$. hyosynoviae indicated by the enzyme-linked immunosorbent assay, complement fixation and indirect hemagglutination tests. Can J Comp Med 1984, 48, 202-207.

9. Friis NF. Some recommendations concerning primary isolation of Mycoplasma suipneumoniae and Mycoplasma flocculare a survey. Nord Vet Med 1975, 27, 337-339.

10. Kobayashi H, Morozumi T, Miyamoto C, Shimizu M, Yamada S, Ohashi S, Kubo M, Kimura K, Mitani K, Ito N, Yamamoto K. Mycoplasma hyorhinis infection levels in lungs of piglets with porcine reproductive and respiratory syndrome (PRRS). J Vet Med Sci 1996, 58, 109-113.

11. Kobisch M, Friis NF. Swine mycoplasmoses. Rev Sci Tech 1996, 15, 1569-1605.

12. Kurth KT, Hsu T, Snook ER, Thacker EL, Thacker BJ, Minion FC. Use of a Mycoplasma hyopneumoniae nested polymerase chain reaction test to determine the optimal sampling sites in swine. J Vet Diagn Invest 2002, 14, 463-469.

13. Lin JH, Chen SP, Yeh KS, Weng CN. Mycoplasma hyorhinis in Taiwan: diagnosis and isolation of swine pneumonia pathogen. Vet Microbiol 2006, 115, 111-
116.

14. Liu W, Fang L, Li S, Li Q, Zhou Z, Feng Z, Luo R, Shao G, Wang L, Chen H, Xiao S. Complete Genome Sequence of Mycoplasma hyorhinis Strain HUB-1. J Bacteriol 2010, 192, 5844-5845.

15. Ross RF. Mycoplasmal diseases. In: Leman AD, Straw BE, Mengeling WL, D'Allaire S, Taylor DJ (eds.). Diseases of Swine. 7th ed. pp. 537-551, Iowa State University Press, Iowa, 1992.

16. Sibila M, Pieters M, Molitor T, Maes D, Haesebrouck F, Segalés J. Current perspectives on the diagnosis and epidemiology of Mycoplasma hyopneumoniae infection. Vet J 2009, 181, 221-231.

17. Stakenborg T, Vicca J, Butaye $\mathbf{P}$, Imberechts $\mathbf{H}$, Peeters J, de Kruif A, Haesebrouck F, Maes D. A multiplex PCR to identify porcine mycoplasmas present in broth cultures. Vet Res Commun 2006, 30, 239-247.

18. Stärk KD, Nicolet J, Frey J. Detection of Mycoplasma hyopneumoniae by air sampling with a nested PCR assay. Appl Environ Microbiol 1998, 64, 543-548.

19. Taschke C, Klinkert MQ, Wolters J, Herrmann R. Organization of the ribosomal RNA genes in Mycoplasma hyopneumoniae: the 5S rRNA gene is separated from the $16 \mathrm{~S}$ and $23 \mathrm{~S}$ rRNA genes. Mol Gen Genet 1986, 205, 428-433.

20. Thacker EL. Mycoplasmal disease. In: Straw BE, Zimmerman JJ, D'Allaire S, Taylor DJ (eds.). Diseases of Swine. 9th ed. pp. 701-717, Wiley-Blackwell, Iowa, 2006.

21. Thacker EL. Diagnosis of Mycoplasma hyopneumoniae. Anim Health Res Rev 2004, 5, 317-320.

22. Verdin E, Saillard C, Labbé A, Bové JM, Kobisch M. A nested PCR assay for the detection of Mycoplasma hyopneumoniae in tracheobronchiolar washings from pigs. Vet Microbiol 2000, 76, 31-40.

23. Walsh PS, Erlich HA, Higuchi R. Preferential PCR amplification of alleles: mechanisms and solutions. Genome Res 1992, 1, 241-250. 